\title{
VARIATIONS ON A CONJECTURE OF HALPERIN
}

\author{
GREGORY LUPTON \\ Department of Mathematics, Cleveland State University \\ Cleveland, Ohio 44115, U.S.A. \\ E-mail: Lupton@math.csuohio.edu
}

\begin{abstract}
Halperin has conjectured that the Serre spectral sequence of any fibration that has fibre space a certain kind of elliptic space should collapse at the $E_{2}$-term. In this paper we obtain an equivalent phrasing of this conjecture, in terms of formality relations between base and total spaces in such a fibration (Theorem 3.4). Also, we obtain results on relations between various numerical invariants of the base, total and fibre spaces in these fibrations. Some of our results give weak versions of Halperin's conjecture (Remark 4.4 and Corollary 4.5). We go on to establish some of these weakened forms of the conjecture (Theorem 4.7). In the last section, we discuss extensions of our results and suggest some possibilities for future work.
\end{abstract}

1. Introduction. We begin with a description of the conjecture referred to in the title. In this paper, all spaces are simply connected CW complexes and are of finite type over $\mathbb{Q}$, i.e., have finite-dimensional rational homology groups. A fibration $F \stackrel{j}{\longrightarrow} E \stackrel{p}{\longrightarrow}$ $B$ is said to be totally non-cohomologous to zero (abbreviated TNCZ) if the induced homomorphism $j^{*}: H^{*}(E ; \mathbb{Q}) \rightarrow H^{*}(F ; \mathbb{Q})$ is onto. This is a very strong condition to place on a fibration. It is equivalent to requiring that the Serre spectral sequence (for cohomology with rational coefficients) collapse at the $E_{2}$-term (cf. [McC,Th.5.9]). In this case there is an isomorphism $H^{*}(E ; \mathbb{Q}) \cong H^{*}(B ; \mathbb{Q}) \otimes H^{*}(F ; \mathbb{Q})$ of $H^{*}(B ; \mathbb{Q})$-modules. Thus a TNCZ fibration is somewhere between being trivial from the rational homology point of view and being trivial from the rational cohomology algebra point of view (cf. Example 1.2).

In the sequel we focus on certain fibre spaces $F$ that satisfy the following conditions:

(1) $H^{*}(F ; \mathbb{Q})$ is finite-dimensional.

(2) $\pi_{*}(F) \otimes \mathbb{Q}$ is finite-dimensional.

1991 Mathematics Subject Classification: Primary 55P62.

This paper, which is in final form and which will not appear elsewhere, was written whilst the author was a guest at the Max-Planck-Institut für Mathematik. The work was begun whilst the author was a visitor at the Université Catholique de Louvain. Thanks to both institutions for their support. 
(3) The Euler characteristic of $F$, i.e., $\sum_{i}(-1)^{i} \operatorname{dim}\left(H^{i}(F ; \mathbb{Q})\right)$, is positive.

A space that satisfies (1) and (2) is called (rationally) elliptic. See [Ha $]$, [Fé,Ch.5] or $[\mathrm{Au}]$ for a discussion of these spaces. It is known that elliptic spaces have non-negative Euler characteristic $\left[\mathrm{Ha}_{1}\right]$. So condition (3) further restricts $F$ to being one of two types of elliptic space. We often refer to a space that satisfies conditions (1)-(3) as a positively elliptic space. However, we also refer to such spaces in the long-hand, as 'elliptic with positive Euler characteristic', particularly when stating results.

The conjecture of the title, with which we are concerned, is as follows:

1.1. Conjecture (Halperin). Let $F$ be elliptic with positive Euler characteristic. Then any fibration $F \rightarrow E \rightarrow B$ is TNCZ.

This conjecture has been established in various cases, but in general it remains open. Some results that concern it are mentioned later in the introduction.

We point out that this paper does not resolve Conjecture 1.1, not even in special cases! Rather, as the title suggests, we are concerned here with variations on the theme provided by the conjecture. These variations come about by considering consequences of Conjecture 1.1, assuming it to be true. The motivation is two-fold: First, it is hoped to open up new lines of approach to the conjecture itself. Second, by looking at such consequences one can obtain weak versions of the conjecture. On the one hand, these weak versions might prove more tractable than the original. On the other hand, they should lead to a fuller understanding of the conjecture.

Although we consider consequences of Conjecture 1.1, some of our results are independent of the status of this conjecture and furthermore are interesting in their own right. For instance, Theorem 4.7 specializes to obtain the following result: If $F \rightarrow E \rightarrow$ $S^{2 n+1}$ is a fibration with fibre a positively elliptic space and base an odd sphere, then $\operatorname{cat}_{0}(E)=\operatorname{cat}_{0}(F)+1$. This result establishes a weak form of Conjecture 1.1. But also, for instance, it can be viewed as a strong form of Ganea's conjecture in the (very) restricted circumstances to which it applies.

Next, we outline the contents of the paper. This introductory section continues with a discussion of positively elliptic spaces and some of their properties. We go on to discuss models of rational fibrations, the main technical tool that we use. The introduction finishes with a brief summary of some results on Conjecture 1.1 and some notational conventions. Section 2 is a short technical section, although in Theorem 2.2 we obtain a very strong consequence of Conjecture 1.1. In Section 3 we relate the formality of $E$ and $B$, for a class of fibrations $F \rightarrow E \rightarrow B$ including those to which Conjecture 1.1 applies. In Proposition 3.2, for example, we show $B$ formal implies $E$ formal, under the hypothesis that Conjecture 1.1 is true. We also obtain an equivalent phrasing of Conjecture 1.1, in Theorem 3.4. In Section 4 we consider some numerical rational homotopy invariants. Under the hypothesis that Conjecture 1.1 is true, we obtain inequalities that relate the values of these invariants on base, total and fibre spaces of a suitable fibration (Remark 4.4). These inequalities can therefore be viewed as weak versions of Halperin's conjecture. We go on to establish these weakened forms of the conjecture in certain restricted circumstances: For some of the invariants, we obtain complete results in case the base 
space is a wedge of odd-dimensional spheres (Theorem 4.7). In the last section, we discuss extensions of our earlier results. Here we suggest various directions for future work, in part by offering specific questions on these topics.

The spaces $F$ that feature in Conjecture 1.1 are clearly of a very restricted kind. We continue with a discussion of some of their properties. We have characterized them by conditions (1)-(3) above. However, results of Halperin [Ha $]$ allow for various characterizations. Halperin shows that, for an elliptic space $F$, the three conditions of positive Euler characteristic, $\chi_{\pi}(F)=0$ and $H^{\text {odd }}(F ; \mathbb{Q})=0$ are equivalent. Here, $\chi_{\pi}(F)$ denotes the so-called homotopy Euler characteristic of $F$. This is a number defined for any space that has finite-dimensional rational homotopy by $\chi_{\pi}(F):=\sum_{i}(-1)^{i} \operatorname{dim}\left(\pi_{i}(F) \otimes \mathbb{Q}\right)$. The cohomology algebra of a positively elliptic space $F$ is zero in odd degrees and has a presentation of the form

$$
H^{*}(F ; \mathbb{Q}) \cong \frac{\mathbb{Q}\left[x_{1}, \ldots, x_{n}\right]}{\left(R_{1}, \ldots, R_{n}\right)}
$$

with relations generated by a maximal regular sequence $\left\{R_{1}, \ldots, R_{n}\right\}$ in the polynomial algebra $\mathbb{Q}\left[x_{1}, \ldots, x_{n}\right]$. Here, the relations $R_{1}, \ldots, R_{n}$ need not be homogeneous (length) polynomials. The minimal model of such a space is of a particularly restricted form. Recall that the minimal model of a space $X$ is a differential graded (henceforth DG) algebra $\mathcal{M}_{X}, d_{X}$, that as a graded algebra is a free graded commutative algebra (polynomial on even degree generators and exterior on odd degree generators). Also, its (degree +1$)$ differential $d_{X}$ is decomposable, in the sense that it induces the trivial differential after passing to the quotient module of indecomposables, i.e., it has zero linear part. See [Gr$\mathrm{Mo}],\left[\mathrm{Ha}_{3}\right]$ and $[\mathrm{Ta}]$ for the basics of minimal models and their use in rational homotopy theory. The book by Félix [Fé] contains more recent material and references. Condition (1) above implies that the minimal model has finite-dimensional cohomology, as the cohomology of the minimal model is identified with that of the space. Condition (2) translates into the condition that the minimal model be finitely-generated as a free graded algebra, since the algebra generators of the minimal model are identified, as a graded vector space, with the rational homotopy groups of the space. For an elliptic space, condition (3) greatly restricts the form of the minimal model further. It implies, for instance, that up to isomorphism the model is a pure model [ $\left.\mathrm{Ha}_{1}\right]$. This is to say that it has the form

$$
\mathcal{M}_{F}, d_{F}=\Lambda\left(V^{\text {even }}\right) \otimes \Lambda\left(V^{\text {odd }}\right), d_{F}
$$

with $d_{F}\left(V^{\text {even }}\right)=0$ and $d_{F}\left(V^{\text {odd }}\right) \subseteq \Lambda\left(V^{\text {even }}\right)$. For an elliptic space, condition (3) further implies that the minimal model has the same number of even degree generators as odd degree generators. In symbols, this means $\operatorname{dim}\left(V^{\text {even }}\right)=\operatorname{dim}\left(V^{\text {odd }}\right)$ and this fact corresponds to the condition that $\chi_{\pi}(F)=0$. We state one more property of these remarkable spaces. We have said that the cohomology algebra of a positively elliptic space is zero in odd degrees. In fact, more is true. Suppose $\Lambda V, d$ is any pure model, as above. We place a second grading on $\Lambda V$ by setting $(\Lambda V)_{k}=\Lambda V^{\text {even }} \otimes \Lambda^{k} V^{\text {odd }}$ for $k \geq 0$. Since $d\left(V^{\text {even }}\right)=0$ and $d\left(V^{\text {odd }}\right) \subseteq \Lambda\left(V^{\text {even }}\right)$, the differential $d$ decreases second degree by 1 and so $\Lambda V, d$ is a bigraded DG algebra. This second grading passes to cohomology. Now, if $\Lambda V$ is the model of a positively elliptic space, then we have $H_{+}(\Lambda V, d)=0$ (see $\left[\mathrm{Ha}_{1}, \mathrm{Th} .2\right]$ ). 
It is worthwhile remarking that, despite the highly restricted form of $F$, there are many examples of such spaces, some of which correspond to spaces familiar to topologists and geometers: Even-dimensional spheres, complex projective spaces, Grassmann manifolds and in general homogeneous spaces $G / H$ with $G$ a compact, connected Lie group and $H$ a closed subgroup of maximal rank are all examples of positively elliptic spaces. Further, given any algebra presented as above, there is some space $F$, necessarily positively elliptic, that realizes the algebra as its rational cohomology algebra.

Next, we survey some material on rational fibrations and their minimal models. For a fuller discussion see $\left[\mathrm{Ha}_{2}\right]$ or [Fé]. Consider a sequence of DG algebra maps of the form

$$
B, d_{B} \stackrel{i}{\rightarrow} B \otimes \Lambda V, D \stackrel{q}{\rightarrow} \Lambda V, d
$$

in which $i: B, d_{B} \rightarrow B \otimes \Lambda V, D$ is the inclusion and $q: B \otimes \Lambda V, D \rightarrow \Lambda V$, $d$ is the projection onto the quotient DG algebra of $B \otimes \Lambda V$ by the ideal generated by $B^{+}$. This sequence is called a KS-extension of $B, d_{B}$ (for Koszul-Sullivan) if there is a well-ordered basis $\left\{v_{\alpha}\right\}_{\alpha \in I}$ of $V$ such that, for each $\alpha \in I, D\left(1 \otimes v_{\alpha}\right) \in B \otimes \Lambda\left(V_{<\alpha}\right)$. Here $V_{<\alpha}$ denotes the subspace of $V$ generated by basis elements $\left\{v_{\beta} \mid \beta<\alpha\right\}$. Such a well-ordered basis is referred to as a KS-basis for the extension. In all cases of interest to this paper, the quotient $\Lambda V, d$ is minimal in the sense described earlier, i.e., it is free with decomposable differential.

Next consider a map $p: E \rightarrow B$ of 1 -connected spaces. There is a corresponding DG algebra map of minimal models, $\mathcal{M}(p): \mathcal{M}_{B}, d_{B} \rightarrow \mathcal{M}_{E}, d_{E}$. Now any map of DG algebras can be converted into a KS-extension [Fé,p.26]. This process is analogous to that of writing a map of spaces as a fibration up to equivalence, and results in the following diagram:

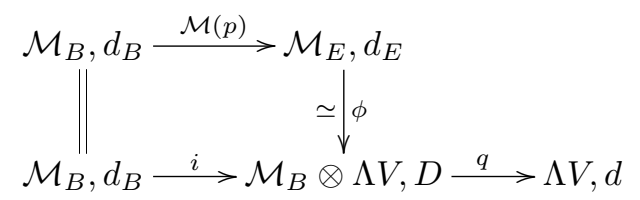

Here, the DG algebra map $\phi$ is a quasi-isomorphism, i.e., it induces an isomorphism on cohomology. A fundamental result of rational homotopy theory asserts that if $p: E \rightarrow B$ is a fibration of 1 -connected spaces with fibre $F$, then $\Lambda V, d$ is a minimal model of the fibre (cf. $\quad\left[\mathrm{Ha}_{2}, \mathrm{Th} .4 .6\right]$ or [Fé,Th.2.3.3]). More generally, we call a sequence of 1-connected spaces $F \rightarrow E \rightarrow B$ a rational fibration if, after forming the KS-model $\mathcal{M}_{B}, d_{B} \rightarrow \mathcal{M}_{B} \otimes \Lambda V, D \rightarrow \Lambda V, d$ of $E \rightarrow B$, the quotient $\Lambda V, d$ is a minimal model of $F$. In this case, we refer to the KS-extension $\mathcal{M}_{B}, d_{B} \rightarrow \mathcal{M}_{B} \otimes \Lambda V, D \rightarrow \Lambda V$, $d$ as the minimal model of the rational fibration. Note that the differential $D$ may have a non-trivial linear part, even though we use the terminology 'minimal' for such a model (cf. Example 1.2 below). Now suppose $F \rightarrow E \rightarrow B$ is a rational fibration with minimal model $\mathcal{M}_{B}, d_{B} \rightarrow \mathcal{M}_{B} \otimes \Lambda V, D \rightarrow \Lambda V, d$. Then the 'fibre inclusion' $j: F \rightarrow E$ is modeled by the projection $q: \mathcal{M}_{B} \otimes \Lambda V, D \rightarrow \Lambda V, d$ and the 'fibration' $p: E \rightarrow B$ by the inclusion $i: \mathcal{M}_{B}, d_{B} \rightarrow \mathcal{M}_{B} \otimes \Lambda V, D$. In particular, it follows from the fundamental result about minimal models that $j^{*}$ is surjective if and only if $q^{*}$ is surjective. 
It is convenient to allow for some flexibility in modeling a given fibration. We say two $K S$-extensions are quasi-isomorphic if there is a commutative diagram

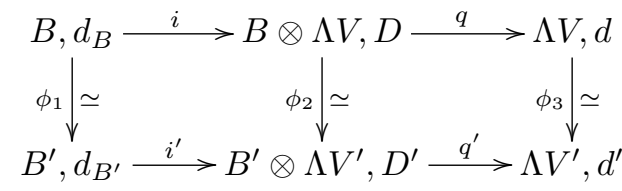

in which each $\phi_{i}$ is a quasi-isomorphism. Notice that in this case, $q^{\prime}$ is surjective if and only if $q$ is surjective, so either KS-extension would be sufficient for determining whether $j^{*}$ is surjective. By a model for a rational fibration, we mean any KS-extension that is quasi-isomorphic to the minimal model.

There is a standard technique for changing the model of a fibration, known as change of $K S$-basis. We mention it here for the sake of reference. Suppose that $B, d_{B} \rightarrow B \otimes \Lambda V, D \rightarrow$ $\Lambda V, d$ is a KS-extension with KS-basis $\left\{v_{\alpha}\right\}$. Suppose given elements $\eta_{\alpha} \in B^{+} \otimes \Lambda\left(V_{<\alpha}\right)$, for each $\alpha \in I$. Define a map of algebras $\phi: B \otimes \Lambda V \rightarrow B \otimes \Lambda V$ by setting $\phi=\iota$ on $B$, and $\phi\left(v_{\alpha}\right)=v_{\alpha}+\eta_{\alpha}$ on basis elements of $V$, then extending to an algebra map. Finally, define a new differential $D^{\prime}$ on $B \otimes \Lambda V$ by $D^{\prime}=\phi^{-1} D \phi$. Then we have an isomorphism of KS-extensions

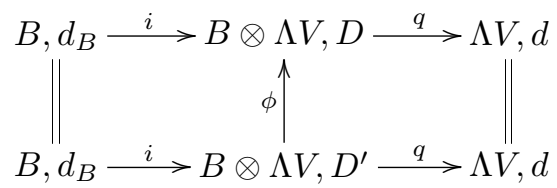

In practice, the new differential $D^{\prime}$ will have some simpler form, or display some desired property. For instance, if we change basis in such a way that $v_{\alpha}+\eta_{\alpha}$ is a $D$-cocycle for some $\alpha$, then $D^{\prime}\left(v_{\alpha}\right)=0$. Another way of obtaining different models for a fibration is to use the pushout in the context of KS-extensions. Suppose we have a KS-extension $B, d_{B} \rightarrow B \otimes \Lambda V, D \rightarrow \Lambda V, d$ and a map of DG algebras $\phi: B, d_{B} \rightarrow B^{\prime}, d_{B^{\prime}}$. We form the pushout as described in [Ba,p.66], for example. (In Baues' terminology, the inclusion $i$ is a cofibration.) This gives the following pushout diagram:

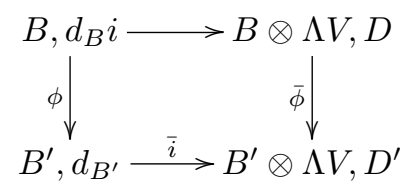

If $\phi$ is a quasi-isomorphism, then so is $\bar{\phi}$. Also, the new differential $D^{\prime}$ projects to the original $d$ on $\Lambda V$. Hence, if $\phi$ is a quasi-isomorphism, we obtain a quasi-isomorphism of KS-extensions as follows:

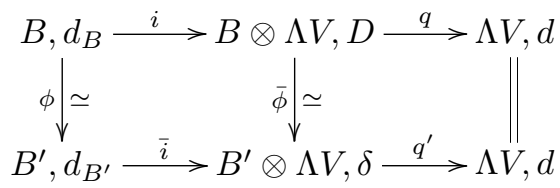

We illustrate the foregoing discussion of models with an example: 
1.2. Example. There is a fibration $S^{2} \rightarrow \mathbb{C} P^{3} \rightarrow S^{4}$ (obtained from the Hopf fibration) which has minimal model

$$
\Lambda\left(w_{4}, w_{7}\right), d_{B} \rightarrow \Lambda\left(w_{4}, w_{7}\right) \otimes \Lambda\left(v_{2}, v_{3}\right), D \rightarrow \Lambda\left(v_{2}, v_{3}\right), d .
$$

Here, subscripts on generators indicate their degree and the differentials are given by $d_{B}\left(w_{4}\right)=0, d_{B}\left(w_{7}\right)=w_{4}^{2}, D\left(v_{2}\right)=0$ and $D\left(v_{3}\right)=v_{2}^{2}-w_{4}$. We obtain $d$ by projecting onto $\Lambda\left(v_{2}, v_{3}\right)$. Notice that $D$ has a non-trivial linear part. This simple example turns out to have several interesting features that illustrate quite well the topics of this paper. In particular, we observe that this fibration is TNCZ but the cohomology of $\mathbb{C} P^{3}$ is not isomorphic as an algebra to the tensor product of the cohomology algebras of $S^{4}$ and $S^{2}$.

The model of a rational fibration is the main technical tool that is used in this paper. Indeed, the results boil down to algebraic results about KS-extensions, which are proven by direct analysis of the model. When we say 'fibration' in the sequel, therefore, we mean rational fibration, as it is to this class of maps that our methods apply.

We now mention two ways to re-phrase Conjecture 1.1 that are used in the sequel. If $F$ is positively elliptic, then its minimal model is pure. We extend the notion of pureness to fibrations with fibre $F$ as follows:

1.3. Definition. Let $F \rightarrow E \rightarrow B$ be a fibration in which $F$ is elliptic with positive Euler characteristic. The fibration is pure (as a fibration) if it has minimal model $\mathcal{M}_{B}, d_{B} \rightarrow \mathcal{M}_{B} \otimes \Lambda V, D \rightarrow \Lambda V, d$ in which $D\left(V^{\text {even }}\right)=0$ and $D\left(V^{\text {odd }}\right) \subseteq \mathcal{M}_{B} \otimes \Lambda\left(V^{\text {even }}\right)$.

Our first rephrasing of Conjecture 1.1 is given in the following:

1.4. TheOREM $\left[\mathrm{Th}_{1}\right]$. For a given fibration $F \rightarrow E \rightarrow B$, in which $F$ is elliptic with positive Euler characteristic, the following are equivalent:

(1) The fibration is TNCZ.

(2) The fibration is pure.

Halperin's conjecture is therefore equivalent to the conjecture that each fibration with fibre a positively elliptic space is a pure fibration. In $\left[\mathrm{Th}_{1}\right]$, Thomas uses his result to show the conjecture is true if the model for $F$ has $\operatorname{dim}\left(V^{\text {even }}\right)=1$ or 2 .

Another re-phrasing of Conjecture 1.1 is given by Meier:

1.5. THEOREM [Me]. Let $F$ be elliptic with positive Euler characteristic. Then the following are equivalent:

(1) Each fibration $F \rightarrow E \rightarrow B$, for arbitrary base space B, is TNCZ.

(2) Each fibration $F \rightarrow E \rightarrow S^{2 n+1}$, for $n \geq 1$, is TNCZ.

(3) The (graded Lie algebra of) negative-degree derivations of the cohomology algebra of $F$ are trivial, $\operatorname{Der}^{<0} H^{*}(F ; \mathbb{Q})=0$.

In [Me], Meier uses his result to establish some special cases of the conjecture.

In addition to these results, various special cases of the conjecture have been established. In [Sh-Te], the conjecture is shown to be true for $F$ a homogeneous space of the form $G / H$, where $G$ is a compact, connected Lie group and $H$ is a closed subgroup of maximal rank. In $[\mathrm{Lu}]$, the result of Thomas is extended to the case when $\operatorname{dim}\left(V^{\text {even }}\right)=3$. 
In $[\mathrm{Ma}]$, it is shown that the class of spaces for which Halperin's conjecture is true is closed under fibrations. This paper of Markl also contains an introduction to Halperin's conjecture and some other interesting results. The conjecture has an interpretation in terms of the rational homotopy theory of $\operatorname{aut}_{1}(F)$, the identity component of the monoid of self-equivalences of $F$. This is an interesting aspect of the conjecture with which, however, we are not directly concerned here. See the articles [Fé-Th] and [Me] for information and references about this.

We end this introductory section with some notation and conventions. In general, we adopt the notation of [Fé] or [Ha-St]. We use $V$ or $W$ to denote a positively graded, rational graded vector space of finite type. We have already used $\Lambda V$ to denote the free graded commutative algebra on the graded vector space $V$. We write $\Lambda^{+} V$ to denote the elements of positive degree. In this paper $\Lambda^{+} V$ is the augmentation ideal of the canonical augmentation of $\Lambda V$. Also, $\Lambda^{n} V$ denotes the vector space of polynomials of homogeneous degree $n$, with respect to the grading by word length. If $V$ is zero in even degrees, for example, this agrees with the $n$ 'th exterior power of $V$. Then $\Lambda^{\geq n} V$ denotes the ideal in $\Lambda V$ generated by $\Lambda^{n} V$. The cohomology of a DG algebra $A, \delta$ is denoted $H(A, \delta)$ or just $H(A)$. The elements of positive degree are denoted $H^{+}(A)$. Other notation and definitions will be given in the sequel.

Acknowledgements. I thank Octav Cornea, Yves Félix, John Oprea and JeanClaude Thomas for several very helpful conversations about this work.

2. Two preliminary results. We present two technical results about models of the fibrations with which we are concerned. In these results, we focus on the case in which the base is a wedge of odd-dimensional spheres or a single such sphere. From Meier's result, cited as Theorem 1.5, these fibrations are of particular interest from our point of view.

The following result is not new (cf. [Lu]), but is included for completeness' sake.

2.1. Lemma. Let $F \rightarrow E \rightarrow S^{2 n+1}$ be a fibration in which $F$ is elliptic with positive Euler characteristic. Up to isomorphism, the minimal model $\Lambda(u) \rightarrow \Lambda(u) \otimes \Lambda V, D \rightarrow$ $\Lambda V, d$ has $D$ decomposable and $D\left(V^{\text {even }}\right) \subseteq u \cdot \Lambda^{+}\left(V^{\text {even }}\right)$.

Pr o of. Suppose $\Lambda(u) \rightarrow \Lambda(u) \otimes \Lambda V, D \rightarrow \Lambda V, d$ is the minimal model, with $|u|=2 n+$ 1. The differential $D$ is decomposable by $\left[\mathrm{Ha}_{2}\right.$,Th.1.4(iii)] (cf. Prop.4.12 and Rem.4.18 of the same reference). Now let $v \in V^{\text {even }}$ and write $D(v)=u \chi_{0}+u \chi_{+}$, with $\chi_{0} \in\left(\Lambda^{+} V\right)_{0}$ and $\chi_{+} \in(\Lambda V)_{+}$. The subscripts refer to the second grading of $\Lambda V$ mentioned in the introduction. Applying $D$ again, we obtain $0=D^{2}(v)=-u d\left(\chi_{0}\right)-u d\left(\chi_{+}\right)=-u d\left(\chi_{+}\right)$. Therefore, $\chi_{+}$is a $d$-cocycle in $(\Lambda V)_{+}$. As stated in the introduction, each cocycle of positive second degree is a boundary, so there is an element $\eta \in \Lambda V$ with $d \eta=\chi_{+}$. Hence $D(u \eta)=-u \chi_{+}$and we have $D(v+u \eta)=u \chi_{0}$.

Now use the change of KS-basis argument mentioned in the introduction, replacing each KS-basis element $v \in V^{\text {even }}$ with $v+u \eta$. In the isomorphic KS-extension obtained as a result, we have $D^{\prime}(v)=u \chi_{0}$, as is easily checked. 
The next result shows that Halperin's conjecture implies the strongest possible restriction on a fibration with base a wedge of odd spheres. It uses the fact that a wedge of spheres is a formal space. Recall that a space is formal if its minimal model is determined by its cohomology algebra. Specifically, $X$ is formal if there is a quasiisomorphism

$$
\psi: \mathcal{M}_{X}, d_{X} \rightarrow H^{*}(X ; \mathbb{Q}), 0
$$

We mention in passing that there are many interesting examples of formal spaces including $H$-spaces and co- $H$-spaces, symmetric spaces and simply connected, compact Kähler manifolds (for this last assertion, see [D-G-M-S]). A product or wedge of formal spaces is again a formal space. A positively elliptic space is a formal space. For further discussion of formal spaces, see Section 3.

2.2. TheOREM. Let $F \rightarrow E \rightarrow B$ be a fibration in which $F$ is elliptic with positive Euler characteristic and $B$ is rationally a wedge of odd spheres. If the fibration is TNCZ, then $E \simeq_{\mathbb{Q}} B \times F$ and the fibration (rationally) is trivial.

Proof. Suppose $\Lambda W, d_{B} \rightarrow \Lambda W \otimes \Lambda V, D \rightarrow \Lambda V, d$ is the minimal model of the fibration. By the result of Thomas, cited as Theorem 1.4, this model can be assumed pure. Since $B$ is rationally a wedge of spheres, it is formal and hence there is a quasiisomorphism $\psi: \Lambda W, d_{B} \rightarrow H(B)$. Now form the pushout and obtain a quasi-isomorphic KS-extension $H(B) \rightarrow H(B) \otimes \Lambda V, D^{\prime} \rightarrow \Lambda V, d$. Since the original KS-extension was pure, it follows directly from the pushout construction that this KS-extension is also pure. The observation that we have such a model also follows from a close reading of Thomas' proof of Theorem 1.4

Now consider the pure model $H(B) \rightarrow H(B) \otimes \Lambda V, D \rightarrow \Lambda V, d$. Let $v \in V^{\text {odd }}$ and let $\left\{b_{i}\right\}$ be a basis for $H^{+}(B)$. Write the differential $D$ as

$$
D(v)=d v+\sum_{i} b_{i} \Omega_{i}(v) .
$$

For parity of degree reasons each $\Omega_{i}(v)$ is an odd-degree element of $\Lambda V$. Since this model is pure, $D(d v)=0$. Therefore, applying $D$ to $(*)$ gives $0=D^{2}(v)=-\sum_{i} b_{i} d \Omega_{i}(v)$. For the last expression, we use the fact that products in $H^{+}(B)$ are trivial. Now we have $d \Omega_{i}(v)=0$ for each $i$, so each $\Omega_{i}(v)$ is a $d$-cocycle of odd degree, hence of positive second degree in the second grading of $\Lambda V$ mentioned in the introduction. However, $H_{+}(\Lambda V)=0$. Therefore, we can choose elements $\eta_{i} \in \Lambda V$ for which $\Omega_{i}(v)=d \eta_{i}$. This gives $D\left(\sum_{i} b_{i} \eta_{i}\right)=-\sum_{i} b_{i} \Omega_{i}(v)$.

Finally, make a change of KS-basis, replacing each KS-basis element $v \in V^{\text {odd }}$ with $v+\sum_{i} b_{i} \eta_{i}(v)$. This gives a quasi-isomorphic KS-extension $H(B) \rightarrow H(B) \otimes \Lambda V, D^{\prime} \rightarrow$ $\Lambda V, d$ in which $D^{\prime}=1 \otimes d$, as is easily checked. The result follows.

2.3. Remark. Notice that we cannot relax the hypothesis on the base to allow evendimensional spheres. Indeed, Example 1.2 is a non-trivial fibration with positively elliptic fibre and base an even-dimensional sphere. For more comments along these lines, see Section 5 . 
3. Formality and TNCZ fibrations. Halperin's conjecture asserts that a fibration is close to trivial if the fibre is positively elliptic. So suppose a fibration has base a formal space and fibre a (formal) positively elliptic space. Then the conjecture asserts that the total space is close to a product of formal spaces and therefore close to being formal. This point of view turns out to yield an equivalent formulation of the conjecture.

A formal space has minimal model that is more highly structured than an arbitrary minimal model. We recall here some properties of the bigraded model that are used in the sequel. See [Ha-St] for a full discussion. The bigraded model of a formal space is a minimal model $\Lambda V, d$ for which the vector space of generators has a second grading, $V=\oplus_{i>0} V_{i}$. This gives $\Lambda V$ the structure of a bigraded algebra. Furthermore, the bigraded model is a bigraded DG algebra in the sense that $d: \Lambda V \rightarrow \Lambda V$ decreases second grading by exactly 1 . In particular, $d\left(V_{0}\right)=0$. The second grading therefore passes to cohomology and, as further properties of the bigraded model, we have $H(\Lambda V, d)=H_{0}(\Lambda V, d)$ and $H_{+}(\Lambda V, d)=0$. There is a quasi-isomorphism - indeed, the bigraded model proper given by

$$
\rho: \Lambda V, d \rightarrow H(\Lambda V, d)=H_{0}(\Lambda V, d)
$$

which maps $V_{+}$to zero and each $v \in V_{0}$ to the class it represents in $H(\Lambda V, d)$. A positively elliptic space is formal and its bigraded model has a simple form. We identify $V_{0}=V^{\text {even }}$ and $V_{1}=V^{\text {odd }}$. There are no generators of second degree $\geq 2$. Notice that this accords with the fact that $H_{+}(\Lambda V, d)=0$, as we stated in the introduction. Following [Ha-St], we adopt the notation $V_{(n)}=\oplus_{i=0}^{n} V_{i}$ and $(\Lambda V)_{(n)}=\oplus_{i=0}^{n}(\Lambda V)_{i}$.

3.1. Proposition. Let $F \rightarrow E \rightarrow B$ be a fibration in which $F$ and $B$ are formal. If the fibration is TNCZ, then there is a model

$$
H(B), 0 \rightarrow H(B) \otimes \Lambda V, D \rightarrow \Lambda V, d,
$$

in which $\Lambda V, d$ is the bigraded model of $F$ and $H(B) \otimes \Lambda V, D$ is filtered in the following sense: Let $V=\oplus_{i \geq 0} V_{i}$ be the second grading of the bigraded model. Then $D\left(V_{0}\right)=0$ and for each $i \geq 1, D\left(\bar{V}_{i}\right) \subseteq H(B) \otimes(\Lambda V)_{(i-1)}$.

Pro of. Since the base and fibre are formal, we can suppose there is a model $H(B) \rightarrow$ $H(B) \otimes \Lambda V, \delta \rightarrow \Lambda V, d$ in which $\Lambda V, d$ is the bigraded model of $F$. Suppose that the fibration is TNCZ. We begin by showing that up to isomorphism, $\delta\left(V_{0}\right)=0$.

Let $v \in V_{0}$, so that $v$ represents a class $[v]$ in $H(\Lambda V)$. By assumption, there is some $\delta$-cocycle $\chi \in H(B) \otimes \Lambda V$ with $q^{*}([\chi])=[v]$. It is easy to see that we may choose the $\delta$-cocycle to be $\chi=v+\beta$ with $\beta \in H^{+}(B) \otimes \Lambda V$. Now use the change of KS-basis argument, replacing each KS-basis element $v \in V_{0}$ by $\chi$. Observe that in the isomorphic model, $H(B) \rightarrow H(B) \otimes \Lambda V, \delta_{0} \rightarrow \Lambda V$, d, we have $\delta_{0}\left(V_{0}\right)=0$.

Now suppose inductively that for some $n \geq 0$, we have a model $H(B) \rightarrow H(B) \otimes$ $\Lambda V, \delta_{n} \rightarrow \Lambda V, d$ in which $\delta_{n}$ is a filtered differential on $H(B) \otimes \Lambda V_{(n)}$, i.e., $\delta_{n}\left(V_{i}\right) \subseteq$ $H(B) \otimes(\Lambda V)_{(i-1)}$ for $i=0, \ldots, n$. Let $v \in V_{n+1}$ and write

$$
\delta_{n}(v)=d v+\xi_{(n)}+\xi_{+}
$$

for elements $\xi_{(n)} \in H^{+}(B) \otimes(\Lambda V)_{(n)}$ and $\xi_{+} \in H^{+}(B) \otimes(\Lambda V)_{\geq n+1}$. 
Claim. There is an element $\eta \in H^{+}(B) \otimes \Lambda V$ for which $\delta_{n}(v)=d v+\xi_{(n)}+\delta_{n}(\eta)$.

Proof of Claim. Suppose that $\xi_{+}=\xi_{+}^{m}+\xi_{+}^{m+1}+\cdots+\xi_{+}^{M}$, for some $2 \leq m \leq M$, with each $\xi_{+}^{i} \in H^{i}(B) \otimes(\Lambda V)_{\geq n+1}$. Let $\left\{b_{j}\right\}$ be a basis for $H^{m}(B)$ and write the 'lowest' term $\xi_{+}^{m}$ as $\xi_{+}^{m}=\sum_{j} b_{j} \otimes \chi_{j}$ for suitable elements $\chi_{j} \in(\Lambda V)_{\geq n+1}$. Applying $\delta_{n}$ to $\left(*_{1}\right)$ gives

$$
0=\delta_{n}^{2}(v)=\delta_{n}\left(d v+\xi_{(n)}\right)+\delta_{n}\left(\xi_{+}^{m}\right)+\sum_{i=m+1}^{M} \delta_{n}\left(\xi_{+}^{i}\right) .
$$

The induction hypothesis on $\delta_{n}$ implies that $\delta_{n}\left(d v+\xi_{(n)}\right) \in H(B) \otimes(\Lambda V)_{(n-1)}$. The ideal in $H(B) \otimes \Lambda V$ generated by elements of $H(B)$ of degree at least $m+1$ is $\delta_{n}$-stable and contains all terms contributed by the $\delta_{n}\left(\xi_{+}^{i}\right)$ for $i \geq m+1$. Therefore, in equation $\left(*_{2}\right)$, terms of the form $\sum_{j}(-1)^{m} b_{j} \otimes d\left(\chi_{j}\right)$ are the only contributions to $H^{m}(B) \otimes(\Lambda V)_{\geq n}$. It follows that each $d\left(\chi_{j}\right)=0$. Since this is the bigraded model, in which $H_{+}(\Lambda V)=0$, there are elements $\eta_{j} \in \Lambda V$ such that $(-1)^{m} \chi_{j}=d\left(\eta_{j}\right)$, for each $j$. Thus we have

$$
\delta_{n}\left(\sum_{j} b_{j} \otimes \eta_{j}\right)=\xi_{+}^{m}+\zeta_{+}^{m+1}+\cdots+\zeta_{+}^{M^{\prime}},
$$

with each $\zeta_{+}^{j} \in H^{j}(B) \otimes(\Lambda V)_{\geq n+1}$, for $m+1 \leq j \leq M^{\prime}$. Substituting this into $\left(*_{1}\right)$ above gives

$$
\delta_{n}(v)=d v+\xi_{(n)}+\xi_{+}^{\prime}+\delta_{n}\left(\sum_{j} b_{j} \otimes \eta_{j}\right),
$$

where $\xi_{+}^{\prime}=\xi_{+}-\xi_{+}^{m}-\sum_{j \geq m+1} \zeta_{+}^{j} \in H^{\geq m+1}(B) \otimes(\Lambda V)_{\geq n+1}$. An induction argument on $m$, repeating this last step as necessary, shows the claim. End of Proof of Claim.

Hence we can make another change of KS-basis, this time replacing a KS-basis element $v \in V_{n+1}$ by $v-\eta$. In the isomorphic model $H(B) \rightarrow H(B) \otimes \Lambda V, \delta_{n+1} \rightarrow \Lambda V, d$ we have $\delta_{n+1}(v)=d v+\xi_{(n)}$, with $\xi_{(n)} \in H^{+}(B) \otimes(\Lambda V)_{(n)}$. Therefore, $\delta_{n+1}$ is a filtered differential on $H(B) \otimes \Lambda V_{(n+1)}$.

To finish, use the inductive step just proved to make a global change of KS-basis, working inductively over $n$. This results in a filtered model as required.

We now develop the main result of the section. The next proposition is a little more general than we need in the sequel. However, it is interesting in its own right. In it, we only assume that the fibre space is formal and elliptic. Recall Halperin's result $\left[\mathrm{Ha}_{1}\right]$, that any elliptic space has non-negative Euler characteristic. If a space is positively elliptic, then it is formal. However, a space that is formal and elliptic need not have positive Euler characteristic. For instance, a product of odd-dimensional spheres is elliptic and formal, but has Euler characteristic equal to zero. There are strong restrictions on a space, however, that follow from the hypothesis of formal and elliptic. We do not dwell on this point here, but simply point out that any space that is formal and elliptic has a two-stage bigraded model (cf. [Fé-Ha $]$ ). This is the feature of these spaces that we use in the result.

3.2. Proposition. Let $F \rightarrow E \rightarrow B$ be a fibration in which $F$ is formal and elliptic and $B$ is formal. If the fibration is TNCZ, then $E$ is formal also. 
Proof. From Proposition 3.1 we have a model of the fibration

$$
H(B), 0 \rightarrow H(B) \otimes \Lambda V, D, \rightarrow \Lambda V, d
$$

in which $D\left(V_{0}\right)=0$ and for $i \geq 1, D\left(V_{i}\right) \subseteq H(B) \otimes(\Lambda V)_{(i-1)}$. This means, in particular, that $D\left(V_{1}\right) \subseteq H(B) \otimes(\Lambda V)_{0}$. Since the bigraded model for $F$ is two-stage, and thus $V=V_{0} \oplus V_{1}$, the total space $H(B) \otimes \Lambda V, D$ in this model is actually a bigraded DG algebra.

We now show that, with respect to this bigrading, $H_{+}(H(B) \otimes \Lambda V)=0$. For let $x \in(H(B) \otimes \Lambda V)_{r}$ be a $D$-cocycle, for any $r \geq 1$. We show that $x$ is exact with an argument similar to that used to show the claim in Proposition 3.1. Write $x$ as $x=x_{m}+x_{m+1}+\cdots+x_{M}$, for $2 \leq m \leq M$, where each $x_{i} \in H^{i}(B) \otimes(\Lambda V) r$. Let $\left\{b_{j}\right\}$ be a basis for $H^{m}(B)$. Then we can write the 'lowest' term $x_{m}$ as $x_{m}=\sum_{j} b_{j} \otimes \chi_{j}$ for appropriate terms $\chi_{j} \in(\Lambda V)_{r}$. Now $(D-d)\left(\chi_{j}\right) \in H^{+}(B) \otimes \Lambda V$ and also the ideal $H^{\geq m+1}(B) \otimes \Lambda V$ is $D$-stable. Therefore, as $x$ is a $D$-cocycle, we have

$$
0=D(x) \equiv(-1)^{m} \sum_{j} b_{j} \otimes d\left(\chi_{j}\right)
$$

where the congruence is modulo the ideal $H^{\geq m+1}(B) \otimes \Lambda V$. Therefore, $d \chi_{j}=0$ and, since $\Lambda V, d$ is the bigraded model and since $r$ is positive, there are elements $\eta_{j}$ with $d \eta_{j}=(-1)^{m} \chi_{j}$ for each $j$. This yields $D\left(\sum_{j} b_{j} \otimes \eta_{j}\right) \equiv \sum_{j} b_{j} \otimes \chi_{j}$, again modulo $H^{\geq m+1}(B) \otimes \Lambda V$. Now we can write

$$
x=D\left(\sum_{j} b_{j} \otimes \eta_{j}\right)+x_{m+1}^{\prime}+\cdots+x_{M^{\prime}}^{\prime},
$$

with each $x_{i}^{\prime} \in H^{i}(B) \otimes(\Lambda V) r$. An induction argument repeating this argument as necessary obtains the result that $x$ is $D$-exact.

We have shown that $H(H(B) \otimes \Lambda V, \delta)=H_{0}(H(B) \otimes \Lambda V, \delta)$. But now the projection

$$
p: H(B) \otimes \Lambda V, \delta \rightarrow \frac{H(B) \otimes \Lambda V, \delta}{\left(\delta V_{1}\right)}=H_{0}(H(B) \otimes \Lambda V, \delta)
$$

is a quasi-isomorphism. Since $H(B) \otimes \Lambda V, \delta$ is quasi-isomorphic to the minimal model for $E$, and also $p$ is a quasi-isomorphism from it to its cohomology, it follows that $E$ is formal.

The next result is something of a converse to Proposition 3.2.

3.3. Proposition. Let $F$ be be elliptic with positive Euler characteristic and let $F \rightarrow$ $E \rightarrow S^{2 n+1}$ be a fibration. If $E$ is formal, then the fibration is TNCZ.

Proof. From Lemma 2.1 the fibration has a model

$$
\Lambda(u) \rightarrow \Lambda(u) \otimes \Lambda V, D \rightarrow \Lambda V, d
$$

in which $\Lambda(u) \otimes \Lambda V, D$ is actually the minimal model of $E$, and $D\left(V_{0}\right) \subseteq u \cdot \Lambda^{+} V_{0}$. We will show that $E$ formal implies $D\left(V_{0}\right)=0$. Recall the characterization of formality given in [D-G-M-S, Th.4.1]. This says that there is a vector space decomposition $\langle u\rangle \oplus V \cong C \oplus N$ with $D(C)=0, D: N \rightarrow \Lambda(u) \otimes \Lambda V$ injective and such that any cocycle in the ideal $I(N)$ of $\Lambda(u) \otimes \Lambda V$ generated by $N$ is exact. Clearly 
$u \in C$, and we show that $V_{0} \subseteq C$, for any such decomposition. For suppose not, so that $D\left(V_{0}\right) \neq 0$. Choose a KS-basis $V_{0}=\left\langle x_{1}, \ldots, x_{n}\right\rangle$. There is at least one of the $x_{i}$ with non-zero differential, so let $r$ be the largest subscript with $D\left(x_{r}\right) \neq 0$. Note that $x_{r} \in N$ in the decomposition. Now $D\left(V_{0}\right)$ is contained in the ideal of $\Lambda(u) \otimes \Lambda V$ generated by elements $\left\{u x_{1}, \ldots, u x_{r-1}\right\}$, which we denote $I_{r-1}$. For parity of degree reasons, $(D-d)\left(V_{1}\right)$ is contained in the ideal generated by $u \cdot V_{1}$. Also, we have $d\left(V_{1}\right) \subseteq \Lambda^{\geq 2} V_{0}$. Let $J$ denote the ideal of $\Lambda(u) \otimes \Lambda V$ generated by $u \cdot V_{1}+\Lambda^{\geq 2} V_{0}$. Then the image of $D$ is contained in the ideal $I_{r-1}+J$. Now $D\left(x_{r}\right)=u \chi$ for some $\chi$ and hence we obtain a $D$-cocycle $u x_{r} \in I(N)$. This cannot be exact, as it is not in the ideal $I_{r-1}+J$. This contradicts the assumptions on the decomposition $C \oplus N$. Therefore, we must have $V_{0} \subseteq C$ and so $D\left(V_{0}\right)=0$. From this it follows easily that the fibration is TNCZ, because then $q^{*}: H(\Lambda(u) \otimes \Lambda V) \rightarrow H(\Lambda V)$ is surjective onto the generators of $H(\Lambda V)$.

Finally, we collect together the preceding results into the main result of the section. As we see, we have obtained an equivalent formulation of Halperin's conjecture.

3.4. TheOREM. Let F be elliptic with positive Euler characteristic. Then the following are equivalent:

(1) Any fibration with fibre $F$ is TNCZ.

(2) For any fibration $F \rightarrow E \rightarrow B$ in which $B$ is formal, $E$ is formal also.

(3) For any fibration $F \rightarrow E \rightarrow S^{2 n+1}$, the total space $E$ is formal.

Pro of. The implication (1) $\Rightarrow(2)$ follows from Proposition 3.2. (2) $\Rightarrow(3)$ is obvious, since spheres are formal spaces. Assume (3). Then Proposition 3.3 implies that each fibration $F \rightarrow E \rightarrow S^{2 n+1}$ is TNCZ. Hence, from Meier's result (Theorem 1.5), any fibration $F \rightarrow E \rightarrow B$ is TNCZ.

4. Numerical invariants. Here we consider some invariants related to the LusternikSchnirelmann category. Recall that this is a numerical homotopy invariant of a space, defined as one less than the smallest number of open sets required to cover the space, when each is contractible in the space. As is usual in rational homotopy theory, we have 'normalised' so that a sphere has category equal to 1 . This invariant and its approximations have been much studied both in ordinary and rational homotopy theory. See [Ja] for a recent survey with many references. Here we focus on four rational homotopy invariants. We define these invariants and include some discussion, before proceeding to the results:

(Rational) Cup-length: This is the nilpotency - as an algebra - of the rational cohomology algebra of a space $X$. It is denoted here $\operatorname{cup}_{0}(X)$. For example we have $\operatorname{cup}_{0}\left(\mathbb{C} P^{n}\right)=n$ for each $n \geq 1$.

(Rational) Toomer's invariant: As in [Fé-Ha $\left.{ }_{2}, \mathrm{Rem} .9 .3\right]$ we describe this invariant as follows: Let $\Lambda V, d$ be the minimal model of $X$. Consider the projection

$$
p_{n}: \Lambda V \rightarrow \frac{\Lambda V}{\Lambda^{\geq n+1} V} .
$$


We obtain our rational invariant, denoted $\mathrm{e}_{0}(X)$, by setting $\mathrm{e}_{0}(X) \leq n$ if $\left(p_{n}\right)^{*}$ is injective. In other words, $\mathrm{e}_{0}(X)$ is the largest $n$ for which some non-zero class in $H(\Lambda V)$ is represented by a cocycle in $\Lambda^{\geq n} V$.

(Rational) Category: This is the Lusternik-Schnirelmann category of the rationalization of $X$. We denote it $\operatorname{cat}_{0}(X)$ and, following [Fé-Ha 2, Th.4.7], we describe it in terms of the minimal model of $X$ : Set $\operatorname{cat}_{0}(X) \leq n$ if the above projection $p_{n}$ makes $\Lambda V$ into a retract of the quotient $\Lambda V / \Lambda^{\geq n+1} V$.

(Rational) Cone-length: This is the least number of steps required to build the rational homotopy type of a space $X$ as a succession of cofibration sequences of rational spaces. It is denoted $\operatorname{cl}_{0}(X)$. Specifically, set $\operatorname{cl}_{0}(X)=0$ if $X \simeq_{\mathbb{Q}} *$ and $\operatorname{cl}_{0}(X)=1$ if $X$ has the rational homotopy type of a wedge of spheres. In general, set $\operatorname{cl}_{0}(X) \leq n$ if there are spaces $X_{1}, A_{1}, \ldots, A_{n-1}$, each of which has the homotopy type of a wedge of rational spheres, and $n-1$ cofibration sequences $A_{i} \rightarrow X_{i} \rightarrow X_{i+1}$, for $i=1, \ldots, n-1$, such that $X_{n} \simeq_{\mathbb{Q}} X$. In $[\mathrm{Co}]$ it is shown that $\operatorname{cl}_{0}(X)$ agrees with the 'homotopical nilpotency' of the minimal model of $X$, i.e., the least $n$ for which the minimal model is quasi-isomorphic to a DG algebra that is of nilpotency $n$ as an algebra. It is in this latter guise that we meet this invariant here.

We have been a little careless in phrasing the above definitions by implicitly assuming these invariants finite. Although the case when one or other of these is infinite does not seem so interesting in our context, it is allowed for, where appropriate, in the following results.

For these invariants, we always have inequalities as follows:

$$
\operatorname{cup}_{0}(X) \leq \mathrm{e}_{0}(X) \leq \operatorname{cat}_{0}(X) \leq \operatorname{cl}_{0}(X) .
$$

In the special case that $X$ is a formal space, all these invariants agree. In this case we will denote their common value $\operatorname{nil}_{0}(X)$. This usage accords with Cornea's homotopical nilpotency in this case. In particular, if $F$ is a positively elliptic space, then it is formal and we have $\operatorname{cup}_{0}(F)=\mathrm{e}_{0}(F)=\operatorname{cat}_{0}(F)=\operatorname{cl}_{0}(F)$, which we denote by $\operatorname{nil}_{0}(F)$.

We mention some examples to illustrate these invariants:

4.1. Examples. If $X=S^{n}$, then $X$ is formal, our four invariants agree and we have $\operatorname{nil}_{0}(X)=1$. If $X=\mathbb{C} P^{n}$, it is likewise formal and $\operatorname{nil}_{0}(X)=n$. Next, suppose $X=S^{2} \vee S^{2} \cup_{\alpha} e^{5}$, where $\alpha=\left[\iota_{1},\left[\iota_{1}, \iota_{2}\right]\right]$, the triple Whitehead product in $\pi_{4}\left(S^{2} \vee S^{2}\right)$. Then we have $\operatorname{cup}_{0}(X)=1$ but $\mathrm{e}_{0}(X)=\operatorname{cat}_{0}(X)=\operatorname{cl}_{0}(X)=2$. A well-known example of Lemaire-Sigrist, developed by Félix-Halperin (cf. [Fé-Ha 2$]$ ), is $X=\left(\mathbb{C} P^{2} \vee S^{2}\right) \cup_{\omega} e^{7}$ for a certain attaching map $\omega$. This space satisfies $\operatorname{cup}_{0}(X)=\mathrm{e}_{0}(X)=2$ whilst $\operatorname{cat}_{0}(X)=$ $\operatorname{cl}_{0}(X)=3$. Furthermore, this example has $\mathrm{e}_{0}\left(X^{n}\right)=2 n$ and $\operatorname{cat}_{0}\left(X^{n}\right)=3 n$. This illustrates that $\mathrm{e}_{0}(X)$ can be smaller than cat ${ }_{0}(X)$ by an arbitrarily large amount.

These invariants behave quite well for products of spaces. The product formula $\operatorname{cup}_{0}(X \times Y)=\operatorname{cup}_{0}(X)+\operatorname{cup}_{0}(Y)$ is well-known. It is easy to see that $\mathrm{e}_{0}$ likewise is additive for products $\left[\mathrm{Fé}-\mathrm{Ha}_{2}, \mathrm{Rem} .9 .3\right]$. Recently, cat ${ }_{0}$ has been shown to be additive for products [Fé-Ha-Le], and $\mathrm{cl}_{0}$ to be additive at least for products of rational Poincaré duality spaces. Indeed, it has been shown that $\mathrm{e}_{0}(X)=\operatorname{cat}_{0}(X)=\operatorname{cl}_{0}(X)$ whenever $X$ is a rational Poincaré duality space (see [Fé-Ha-Le] and [Co-Fé-Le]). Now Halperin's con- 
jecture asserts that in certain fibrations the total space is close to being a product of the base and fibre spaces. These remarks combine to suggest there should be good relations between these invariants for base, total and fibre spaces in such fibrations. We shall see that this is the case.

First we give some results that complement one of Jessup [Je,Prop.3.6]. Let $F \rightarrow$ $E \rightarrow B$ be a TNCZ fibration with $F$ formal. Then Jessup's result gives cat ${ }_{0}(E) \geq$ $\operatorname{cat}_{0}(B)+\operatorname{nil}_{0}(F)$. Actually, his result applies a little more generally and was proved for (what was then thought to be) a different numerical invariant, Mcat ${ }_{0}$. The conclusion for cat $_{0}$ follows by a result of Hess [He], which identified cat ${ }_{0}$ with Mcat $_{0}$.

We specialize this result to the following:

4.2. Proposition. Let $F \rightarrow E \rightarrow B$ be a fibration in which $F$ is elliptic with positive Euler characteristic and $B$ is formal. If the fibration is TNCZ, then $E$ is also formal and we have $\operatorname{nil}_{0}(E) \geq \operatorname{nil}_{0}(B)+\operatorname{nil}_{0}(F)$.

Proof. The formality of $E$ follows immediately from Proposition 3.2. Since $E$ and $B$ are both formal, nil ${ }_{0}=$ cat $_{0}$ for these spaces and the conclusion follows from the result of Jessup mentioned above.

Next, consider the case in which $B$ is not formal. Notice the following result does not require that $F$ be positively elliptic.

4.3. Proposition. Let $F \rightarrow E \rightarrow B$ be a fibration with $F$ formal. If the fibration is $T N C Z$, then we have the following inequalities:

$(1) \operatorname{cup}_{0}(E) \geq \operatorname{cup}_{0}(B)+\operatorname{nil}_{0}(F)$.

(2) $\mathrm{e}_{0}(E) \geq \mathrm{e}_{0}(B)+\operatorname{nil}_{0}(F)$.

Pro of. Actually, for (1) the hypothesis of formality on $F$ is redundant. Recall from the introduction, that if a fibration $F \rightarrow E \rightarrow B$ is TNCZ, then $H^{*}(E ; \mathbb{Q}) \cong H^{*}(B ; \mathbb{Q}) \otimes$ $H^{*}(F ; \mathbb{Q})$ as $H^{*}(B ; \mathbb{Q})$-modules. Under this isomorphism, two elements of $1 \otimes H^{*}(F ; \mathbb{Q})$ multiply as $(1 \otimes x)(1 \otimes y)=1 \otimes x y+\chi$, for some $\chi$ in the ideal generated by $H^{+}(B)$. It follows that $\operatorname{cup}_{0}(E) \geq \operatorname{cup}_{0}(B)+\operatorname{cup}_{0}(F)$.

For (2), we work with a model of the fibration. Suppose that $F$ is formal and the fibration is TNCZ. Then it has a model $\Lambda W, d_{B} \rightarrow \Lambda W \otimes \Lambda V, D \rightarrow \Lambda V, d$ in which $D\left(V_{0}\right)=$ 0 . This follows from the argument in the first two paragraphs of the proof of Proposition 3.1, replacing $H(B)$ there by $\Lambda W$. So let $\alpha \in \Lambda^{\geq n} V$ be a cocycle representative for some non-zero class in $H(\Lambda V)$. Since $F$ is formal, we can suppose that $\alpha \in \Lambda^{\geq n} V_{0}$. In our model we have $D\left(V_{0}\right)=0$, so $[\alpha] \in H(\Lambda W \otimes \Lambda V)$. Furthermore, $\alpha$ is not $D$-exact, since it is not $d$-exact. Let $\beta \in \Lambda^{\geq m} W$ be a cocycle representative for some non-zero class of $H(\Lambda W)$. Since we have $H(\Lambda W \otimes \Lambda V) \cong H(\Lambda W) \otimes H(\Lambda V)$ as $H(\Lambda W)$-modules, the product $[\beta][\alpha]=[\beta \alpha]$ is non-zero in $H(\Lambda W \otimes \Lambda V)$. Now $\alpha \beta \in \Lambda \geq m+n(W \oplus V)$ so $\mathrm{e}_{0}(E) \geq m+n$.

Example 1.2 illustrates that inequality, as in Proposition 4.3, is the best that can be hoped for in general. But see below for stronger relations in special cases.

If $B$ is not formal in Proposition 4.3, there is no a priori reason why the invariants $\operatorname{cup}_{0}, \mathrm{e}_{0}$ and cat ${ }_{0}$ for $B$ or $E$ should agree. Therefore, it can be thought of as giving three 
distinct necessary conditions for Halperin's conjecture to be true. We summarise this in the following:

4.4. Remark. Let $F \rightarrow E \rightarrow B$ be a fibration in which $F$ is elliptic with positive Euler characteristic. If $B$ is formal and if the fibration is TNCZ, then $E$ is also formal and $\operatorname{nil}_{0}(E) \geq \operatorname{nil}_{0}(B)+\operatorname{nil}_{0}(F)$. For general $B$, if the fibration is TNCZ then we have three inequalities

$$
\begin{aligned}
\operatorname{cup}_{0}(E) & \geq \operatorname{cup}_{0}(B)+\operatorname{nil}_{0}(F) \\
\mathrm{e}_{0}(E) & \geq \mathrm{e}_{0}(B)+\operatorname{nil}_{0}(F) \\
\operatorname{cat}_{0}(E) & \geq \operatorname{cat}_{0}(B)+\operatorname{nil}_{0}(F) .
\end{aligned}
$$

Each of these inequalities gives a necessary condition for Halperin's conjecture to be true.

A much stronger consequence follows if we restrict the base as follows:

4.5. Corollary. Let $F \rightarrow E \rightarrow B$ be a fibration in which $F$ is elliptic with positive Euler characteristic and $B$ is rationally a wedge of odd spheres. If the fibration is TNCZ, then $E$ is formal, the invariants $\operatorname{cup}_{0}(E), \mathrm{e}_{0}(E), \operatorname{cat}_{0}(E)$ and $\operatorname{cl}_{0}(E)$ all agree and their common value, $\operatorname{nil}_{0}(E)$, satisfies $\operatorname{nil}_{0}(E)=1+\operatorname{nil}_{0}(F)$.

Proof. This follows from Theorem 2.2.

So far, we have collected together some consequences of Halperin's conjecture. These consequences can be read as weak versions of Halperin's conjecture. We go on to establish some of these weak versions of the conjecture. We can deal quite well with the case in which the base $B$ is a wedge of odd-dimensional spheres.

The next result generalizes part of [Fé-Ha, Th.10.4(iv)].

4.6. Proposition. Let $F \rightarrow E \rightarrow B$ be any fibration in which $F$ is a rational Poincaré duality space and $B$ is a wedge of odd-dimensional spheres. Then $\mathrm{e}_{0}(E) \geq 1+\mathrm{e}_{0}(F)$.

Proof. Suppose $\Lambda W, d_{B} \rightarrow \Lambda W \otimes \Lambda V, D \rightarrow \Lambda V, d$ is the minimal model of the fibration. Observe that $\Lambda W \otimes \Lambda V, D$ must actually be the minimal model of $E$, i.e., the differential $D$ must be decomposable. Indeed, this is the case for any fibration in which $F$ is a space with finite dimensional rational cohomology and $B$ is a wedge of odd-dimensional spheres, as follows from $\left[\mathrm{Ha}_{2}\right.$, Th.1.4(iii)]. Observe further, that since $B$ is a wedge of spheres, it is both formal and coformal. Thus its minimal model $\Lambda W, d_{B}$ is a bigraded model in the sense discussed earlier, and the differential is quadratic, i.e., $d_{B}: W \rightarrow \Lambda^{2} W$. We use these observations in the proof.

Suppose that $\mathrm{e}_{0}(F)=n$. Since $F$ is a Poincaré duality space, the fundamental class of $F$ can be represented by a cocycle $\alpha \in \Lambda^{\geq n} V$ (cf. [Fé,Lem.5.6.1]). Suppose that $u \in W$ is a generator of lowest (odd) degree, so that $d_{B}(u)=0$. Consider the element $u \alpha \in \Lambda^{\geq n+1}(W \oplus V)$. There is no a priori reason why $u \alpha$ should be a $D$-cocycle, but we will show the following:

Claim. There is an element $\eta \in \Lambda^{\geq n+1}(W \oplus V)$, with $\eta \in(\Lambda W)_{+} \otimes \Lambda V$, such that $D(u \alpha+\eta)=0$.

Proof of Claim. We argue by induction, using the second grading of the bigraded model $\Lambda W$. Let $\left\{b_{i, j}\right\}_{j \in J_{i}}$ be a (vector space) basis of $\left(\Lambda^{+} W\right)_{i}$, for each $i \geq 0$. It is 
convenient for our argument to have this basis be a monomial basis, so that each basis element has a certain length. Also, our element $u$ is one of the basis elements $b_{0, j}$, but we denote it $u$ anyway so as to distinguish it.

Write $D(\alpha)=\sum_{i>0, j} b_{i, j} \Omega_{i, j}$, for suitable elements $\Omega_{i, j} \in \Lambda V$. Each term $b_{i, j} \Omega_{i, j} \in$ $\Lambda \geq n+1(W \oplus V)$, as $D$ is decomposable. Then we have

$$
D(u \alpha)=-\sum_{j} u b_{0, j} \Omega_{0, j}-\sum_{i \geq 1, j} u b_{i, j} \Omega_{i, j} .
$$

Now $\Lambda W, d_{B}$ is the minimal model of a wedge of spheres, whose cohomology has trivial products. It follows that each $u b_{0, j} \in\left(\Lambda^{\geq 2} W\right)_{0}$ is a $d_{B}$-cocycle. So $d_{B}\left(\eta_{1, j}\right)=u b_{0, j}$ for some $\eta_{1, j} \in(\Lambda W)_{1}$. Furthermore, since $\Lambda W, d_{B}$ is coformal, each $\eta_{1, j}$ is of length one less than $u b_{0, j}$. For each $j$, we have

$$
D\left(\eta_{1, j} \Omega_{0, j}\right)=u b_{0, j} \Omega_{0, j}+(-1)^{\left|\eta_{1, j}\right|} \eta_{1, j} D\left(\Omega_{0, j}\right) .
$$

From the observation about the length of each $\eta_{1, j}$, together with the fact that $D$ is decomposable, it follows that each $\eta_{1, j} \Omega_{0, j} \in \Lambda^{\geq n+1}(W \oplus V)$ and each $\eta_{1, j} D\left(\Omega_{0, j}\right) \in$ $\Lambda^{\geq n+2}(W \oplus V)$. Finally, write $\eta_{(1)}=\sum_{j} \eta_{1, j} \Omega_{0, j}$, so that $\eta_{(1)} \in \Lambda^{\geq n+1}(W \oplus V)$ and $\eta_{(1)} \in(\Lambda W)_{+} \otimes \Lambda V$. Then we have

$$
D\left(u \alpha+\eta_{(1)}\right)=-\sum_{i \geq 1, j} u b_{i, j} \Omega_{i, j}+\sum_{j}(-1)^{\left|\eta_{1, j}\right|} \eta_{1, j} D\left(\Omega_{0, j}\right)=\sum_{i \geq 1, j} b_{i, j} \Omega_{i, j}^{(1)},
$$

for suitable $\Omega_{i, j}^{(1)} \in \Lambda V$, and each $b_{i, j} \Omega_{i, j}^{(1)} \in \Lambda \geq n+2(W \oplus V)$. This starts the induction.

Now suppose inductively that we have an element $\eta_{(r)} \in \Lambda^{\geq n+1}(W \oplus V)$ with $\eta_{(r)} \in$ $(\Lambda W)_{+} \otimes \Lambda V$ and $D\left(u \alpha+\eta_{(r)}\right)=\sum_{i \geq r, j} b_{i, j} \Omega_{i, j}^{(r)} \in \Lambda \geq n+2(W \oplus V)$. Re-write $\sum_{j} b_{r, j} \Omega_{r, j}^{(r)}$, the part of $D\left(u \alpha+\eta_{(r)}\right)$ that contains terms of lowest second degree in $\Lambda W$, as follows: Let $\left\{c_{k}\right\}_{k \in K}$ be a (vector space) basis for $\Lambda V$. Then write

$$
\sum_{j} b_{r, j} \Omega_{r, j}^{(r)}=\sum_{k} \beta_{r, k} c_{k}
$$

for suitable terms $\beta_{r, k} \in(\Lambda W)_{r}$. For $i \geq r+1$, we have $D\left(b_{i, j} \Omega_{i, j}^{(r)}\right) \in(\Lambda W)_{\geq r} \otimes \Lambda V$. So working modulo the ideal generated by $(\Lambda W)_{\geq r}$ in $\Lambda W \otimes \Lambda V$, we have

$$
0=D^{2}\left(u \alpha+\eta_{(r)}\right) \equiv \sum_{k} d_{B}\left(\beta_{r, k}\right) c_{k}
$$

Hence $d_{B}\left(\beta_{r, k}\right)=0$ for each $k$. Recall once again that $\Lambda W, d_{B}$ is the bigraded model, with $H_{+}\left(\Lambda W, d_{B}\right)=0$. Since $r \geq 1$, it follows that each $\beta_{r, k}$ is $d_{B}$-exact. So $d_{B}\left(\eta_{r+1, k}\right)=\beta_{r, k}$ for some $\eta_{r+1, k} \in(\Lambda W)_{r+1}$. As $\Lambda W$ is coformal, $\eta_{r+1, k} c_{k} \in \Lambda \geq n+1(W \oplus V)$ for each $k$. Now set $\eta_{(r+1)}=\eta_{(r)}-\sum_{k} \eta_{r+1, k} c_{k}$. Note that $\eta_{(r+1)} \in \Lambda^{\geq n+1}(W \oplus V)$ and $\eta_{(r+1)} \in$ $(\Lambda W)_{+} \otimes \Lambda V$. A straightforward check shows that

$$
\begin{aligned}
D\left(u \alpha+\eta_{(r+1)}\right) & =\sum_{i \geq r+1, j} b_{i, j} \Omega_{i, j}^{(r)}-\sum_{k}(-1)^{\left|\eta_{r+1, k}\right|} \eta_{r+1, k} D\left(c_{k}\right) \\
& =\sum_{i \geq r+1, j} b_{i, j} \Omega_{i, j}^{(r+1)}
\end{aligned}
$$


for suitable terms $\Omega_{i, j}^{(r+1)} \in \Lambda V$ with each $b_{i, j} \Omega_{i, j}^{(r+1)} \in \Lambda^{\geq n+2}(W \oplus V)$. This completes the inductive step.

Since $B$ is simply connected, the lowest degree of a generator in $W_{r}$ increases strictly with $r$. Hence, by taking $r$ sufficiently large, we obtain an element $\eta_{(r)}$ as in the claim, with $D\left(u \alpha+\eta_{(r)}\right)=0$. End of Proof of Claim.

We now show that this cocycle is not $D$-exact. Suppose that $D(\zeta+\chi)=u \alpha+\eta$ for $\zeta \in \Lambda V$ and $\chi \in \Lambda^{+} W \otimes \Lambda V$. Then $d(\zeta)=0$. However, $\zeta$ has higher degree than $\alpha$, which represents the fundamental class of $F$. Thus $\zeta=d(a)$ for some $a \in \Lambda V$ so $D(a)=\zeta+\chi^{\prime}$ for some $\chi^{\prime} \in \Lambda^{+} W \otimes \Lambda V$. Write $\chi-\chi^{\prime}=\sum_{i, j} b_{i, j} \chi_{i, j}$. Working modulo the ideal in $\Lambda W \otimes \Lambda V$ generated by $\Lambda^{\geq 2} W_{0}+(\Lambda W)_{+}$, we have $D(\chi+\zeta)=D\left(\chi-\chi^{\prime}\right)=$ $\sum_{j}(-1)^{\left|b_{0, j}\right|} b_{0, j} d\left(\chi_{0, j}\right) \equiv u \alpha$. This implies $\alpha$ is $d$-exact, which is a contradiction since $\alpha$ represents the fundamental class of $F$. Therefore, $u \alpha+\eta$ is a non-exact $D$-cocycle in $\Lambda \geq n+1(W \oplus V)$. The result follows.

Next we give the main result of this section.

4.7. TheOREM. Let $F \rightarrow E \rightarrow B$ be a fibration in which $F$ is elliptic with positive Euler characteristic and $B$ is a wedge of odd-dimensional spheres. Then for $E$ we have $\mathrm{e}_{0}(E)=\operatorname{cat}_{0}(E)=\operatorname{cl}_{0}(E)$ and furthermore these equal nil ${ }_{0}(F)+1$.

P r o of. In fact the proof will display a simple model for $E$ of homotopical nilpotency equal to $1+\operatorname{nil}_{0}(F)$. Let $\rho: \Lambda V, d \rightarrow H(F)$ be the bigraded model for $F$. By construction we have $\rho\left(V_{1}\right)=0$ and hence $\rho\left((\Lambda V)_{+}\right)=0$. Consider the ideal ker $\rho \subseteq \Lambda V$. This is a differential ideal since all boundaries are in $\operatorname{ker} \rho$. Further, it is an acyclic ideal since $\rho$ is a surjective quasi-isomorphism. Since $B$ is formal, the fibration has a model $H(B) \rightarrow H(B) \otimes \Lambda V, D \rightarrow \Lambda V, d$. We claim that the ideal $H(B) \otimes \operatorname{ker} \rho$ of $H(B) \otimes \Lambda V$ is also a differential acyclic ideal. To see this, we argue as follows:

Let $\left\{b_{i}\right\}$ be a basis for $H^{+}(B)$. For any $\chi \in \Lambda V$, we can write

$$
D(\chi)=d \chi+\sum_{i} b_{i} \Omega_{i}(\chi)
$$

and a standard argument shows that this defines derivations $\Omega_{i}$ on $\Lambda V$, , each of negative even degree according as the degree of the $b_{i}$ (cf. the result of Meier, cited as Theorem 1.5). Now for $w \in V_{1}$, we have $D(w)=d w+\sum_{i} b_{i} \Omega_{i}(w)$. For parity of degree reasons we must have $\Omega_{i}(w) \in(\Lambda V)_{+}$. It follows that $\Omega_{i}\left((\Lambda V)_{+}\right) \subseteq(\Lambda V)_{+}$for each derivation $\Omega_{i}$.

Next, if $\chi \in \operatorname{ker} \rho \subseteq \Lambda V$, write $\chi=\chi_{0}+\chi_{+}$where $\chi_{0} \in \Lambda V_{0}$ and $\chi_{+} \in(\Lambda V)_{+}$. Since $\chi \in \operatorname{ker} \rho$, it follows that $\chi_{0} \in \operatorname{ker} \rho$ and hence $\chi_{0}=d \eta$ for some $\eta \in(\Lambda V)_{1}$. Then

$$
\begin{aligned}
D(\chi) & =d\left(\chi_{+}\right)+\sum_{i} b_{i} \Omega_{i}\left(\chi_{0}\right)+\sum_{i} b_{i} \Omega_{i}\left(\chi_{+}\right) \\
& =d\left(\chi_{+}\right)+\sum_{i} b_{i} \Omega_{i}(d \eta)+\sum_{i} b_{i} \Omega_{i}\left(\chi_{+}\right) \\
& =d\left(\chi_{+}\right)+\sum_{i} b_{i} d\left(\Omega_{i}(\eta)\right)+\sum_{i} b_{i} \Omega_{i}\left(\chi_{+}\right) .
\end{aligned}
$$


This term is in $H(B) \otimes \operatorname{ker} \rho$ because $\operatorname{ker} \rho$ contains all boundaries in $\Lambda V$ and also, as remarked earlier, $\Omega_{i}\left(\chi_{+}\right) \in \Omega_{i}\left((\Lambda V)_{+}\right) \subseteq(\Lambda V)_{+} \subseteq \operatorname{ker} \rho$. We have shown that $D(\operatorname{ker} \rho) \subseteq$ $H(B) \otimes \operatorname{ker} \rho$ and since $D=0$ on $H(B)$, it follows that $H(B) \otimes \operatorname{ker} \rho$ is $D$-stable.

Further, suppose $\alpha \in H(B) \otimes \operatorname{ker} \rho$ is a $D$-cocycle. We can write $\alpha=\alpha^{\prime}+\sum_{i} b_{i} \alpha_{i}$ with $\alpha^{\prime}$ and each $\alpha_{i}$ in ker $\rho$. Then $D(\alpha)=0$ implies $d\left(\alpha^{\prime}\right)=0$ and hence $\alpha^{\prime}=d \eta^{\prime}$ for some $\eta^{\prime} \in \operatorname{ker} \rho$, as ker $\rho$ is acyclic. Without loss of generality, we can choose $\eta^{\prime} \in(\Lambda V)_{+}$, as $d\left(V_{0}\right)=0$. Then $D\left(\eta^{\prime}\right)=d\left(\eta^{\prime}\right)+\sum_{i} b_{i} \Omega_{i}\left(\eta^{\prime}\right)$. From an earlier remark, each $\Omega_{i}\left(\eta^{\prime}\right) \in$ $(\Lambda V)_{+} \subseteq \operatorname{ker} \rho$. So we have

$$
\alpha=D\left(\eta^{\prime}\right)+\sum_{i} b_{i}\left(\alpha_{i}-\Omega_{i}\left(\eta^{\prime}\right)\right)=D\left(\eta^{\prime}\right)+\sum_{i} b_{i} \alpha_{i}^{\prime}
$$

for elements $\eta^{\prime}, \alpha_{i}^{\prime} \in \operatorname{ker} \rho$. As all products in $H^{+}(B)$ are trivial, $D(\alpha)=0$ implies each $d\left(\alpha_{i}^{\prime}\right)=0$, so that $\alpha_{i}^{\prime}=d \eta_{i}$ for elements $\eta_{i} \in \operatorname{ker} \rho$. But $D\left(b_{i} \eta_{i}\right)=-b_{i} d\left(\eta_{i}\right)$ and hence $\alpha=D\left(\eta^{\prime}-\sum_{i} b_{i} \eta_{i}\right)$ with $\eta^{\prime}-\sum_{i} b_{i} \eta_{i} \in H(B) \otimes \operatorname{ker} \rho$. This shows $H(B) \otimes \operatorname{ker} \rho$ is an acyclic ideal of $H(B) \otimes \Lambda V$.

To finish, notice that the projection

$$
q: H(B) \otimes \Lambda V \rightarrow \frac{H(B) \otimes \Lambda V}{H(B) \otimes \operatorname{ker} \rho}
$$

is a quasi-isomorphism, as $H(B) \otimes \operatorname{ker} \rho$ is acyclic. If $\operatorname{nil}_{0}(F)=n$, then $\Lambda^{>n} V \subseteq \Lambda^{>n} V_{0}+$ $(\Lambda V)_{+} \subseteq \operatorname{ker} \rho$ and hence the quotient $H(B) \otimes \Lambda V /(H(B) \otimes \operatorname{ker} \rho)$ is a nilpotent DG algebra of length $\leq n+1$, and is quasi-isomorphic to a KS-model for $E$. Therefore, it follows from $[\mathrm{Co}]$ that $\mathrm{cl}_{0}(E) \leq n+1$. From Proposition 4.6, we have $n+1 \leq \mathrm{e}_{0}(E)$. But in general we have $\mathrm{e}_{0}(E) \leq \operatorname{cat}_{0}(E) \leq \mathrm{cl}_{0}(E)$. Hence these three invariants must agree and furthermore must equal $n+1$.

5. Discussion and questions. In this last, somewhat informal, section we discuss the foregoing results and give some additional ones. The intention is to indicate both limits on, and possibilities for, development of the work in Sections 3 and 4 . We also offer some specific questions along these lines. All this is collected under two subheadings, according as the topic relates to Section 3 or Section 4.

5.1. Formality. One can attempt a quite general study of relations between the formality of $F, E$ and $B$ in a fibration (cf. $\left[\mathrm{Th}_{2}\right]$ and [Vi]). It is tempting to think that the very restrictive hypotheses of Proposition 3.2 might be relaxed, whilst keeping the conclusion. However, the following sort of example suggests these hypotheses are actually sharp.

EXAMPLE. There is a rational fibration $S^{2} \vee S^{2} \vee S^{2} \rightarrow E \rightarrow S^{3}$ that is TNCZ in which $E$ is not formal. We omit details of this example, as it is similar to [ $\mathrm{Th}_{2}, \mathrm{Ex}$.III.13] and other examples. We note that the fibre is formal with non-zero cohomology only in even degrees. The fibration we have in mind is actually much closer to being trivial than just TNCZ. It satisfies $H^{*}(E ; \mathbb{Q}) \cong H^{*}(B ; \mathbb{Q}) \otimes H^{*}(F ; \mathbb{Q})$ as algebras and admits a rational section. 
In case a weaker conclusion is acceptable, then of course there are more possibilities. In Proposition 3.2, we can remove the hypothesis of ellipticity on the fibre space, but so far only at the cost of a greatly weakened conclusion, thus:

Proposition. Let $F \rightarrow E \rightarrow B$ be a fibration in which $F$ and $B$ are both formal. If the fibration is TNCZ, then E has spherically-generated cohomology.

The proof of this proposition is omitted. It can be proved with an argument similar to that of Proposition 3.2. The above example also suggests this might be a sharp conclusion, without much more restrictive hypotheses.

It would be satisfying to have a converse of Proposition 3.2. In Proposition 3.3, we have such a result but only for a very special base. We offer the following as a specific question in this area:

QUESTION 1. Let $F \rightarrow E \rightarrow B$ be a fibration in which $F$ is formal and elliptic and $B$ is formal. If $E$ is formal, then is the fibration is TNCZ?

Of course, there are many variations on this type of question to investigate. For more results and examples along these lines, see $\left[\mathrm{Th}_{2}\right]$ and $[\mathrm{Vi}]$.

Returning to Conjecture 1.1, it may be possible to apply Theorem 3.4, together with an appropriate obstruction theory for the formality of $E$ in such a fibration. Such an obstruction theory does exist, and I hope to develop this line of investigation in future work.

5.2. Numerical invariants. The results of Section 4 deal nicely with fibrations $F \rightarrow$ $E \rightarrow B$, in which $F$ is positively elliptic and $B$ is a wedge of odd-dimensional spheres, but only for the invariants $\mathrm{e}_{0}$, cat $_{0}$ and $\mathrm{cl}_{0}$.

Question 2. For a fibration $F \rightarrow E \rightarrow B$, in which $F$ is elliptic with positive Euler characteristic and $B$ is a wedge of odd-dimensional spheres, is $\operatorname{cup}_{0}(E)=1+\operatorname{nil}_{0}(F)$ ?

Of course, if Halperin's conjecture is true, it implies an affirmative answer to Question 2 (Corollary 4.5). It is curious that Question 2 remains unresolved whilst its counterpart for the invariants $\mathrm{e}_{0}$, cat $\mathrm{c}_{0}$ and $\mathrm{cl}_{0}$ is established.

Another 'asymmetry' in the results we have presented is the lack of an inequality for cone-length in Proposition 4.3. This gives our next specific question:

QUESTION 3. Let $F \rightarrow E \rightarrow B$ be a fibration with $F$ formal. If the fibration is TNCZ, then is $\mathrm{cl}_{0}(E) \geq \operatorname{cl}_{0}(B)+\operatorname{nil}_{0}(F)$ ?

As regards extending the results presented in Section 4, we can show at least the following:

Proposition. Let $F \rightarrow E \rightarrow B$ be a fibration in which $F$ is elliptic with positive Euler characteristic and $B$ has $\mathrm{e}_{0}(B)=1$. Then $\mathrm{e}_{0}(E) \geq 1+\operatorname{nil}_{0}(F)$.

Of course, if $\mathrm{e}_{0}(B)=1$, then the base space $B$ is rationally a wedge of spheres and $\mathrm{e}_{0}(B)=\operatorname{cat}_{0}(B)=\operatorname{cl}_{0}(B)=1$. Notice, however, that we allow even dimensional spheres 
and also that we do not have Poincaré duality in either the base or the total spaces. The current proof of this Proposition uses a rather involved reduction argument, similar to that of $\left[\mathrm{Th}_{1}\right]$ or Proposition 4.6 here. This result suggests that the 'next step' might be to investigate one of the following questions:

Question 4 (a). Let $F \rightarrow E \rightarrow B$ be a fibration in which $F$ is elliptic with positive Euler characteristic and $\mathrm{e}_{0}(B)=2$. Is $\mathrm{e}_{0}(E) \geq 2+\operatorname{nil}_{0}(F)$ ?

Question 4 (b). Let $F \rightarrow E \rightarrow B$ be a fibration in which $F$ is elliptic with positive Euler characteristic. Is $\mathrm{e}_{0}(E) \geq 1+\operatorname{nil}_{0}(F)$ ?

In the previous two paragraphs, we focussed on the $\mathrm{e}_{0}$-invariant. Choosing one of the other three invariants gives similar questions to be investigated. There is some overlap between all these questions, because of the results of [Co-Fé-Le] and [Fé-Ha-Le] mentioned earlier: $\mathrm{e}_{0}(X)=\operatorname{cat}_{0}(X)=\operatorname{cl}_{0}(X)$ whenever $X$ is a rational Poincaré duality space. Thus, if $F \rightarrow E \rightarrow B$ is a fibration in which $F$ and $B$ are rational Poincaré duality spaces, then so too is $E$ a rational Poincaré duality space and hence the three invariants agree on each of $F, E$ and $B$. Notice that this observation obtains the first part of the conclusion to Theorem 4.7 in the case $B=S^{2 n+1}$. Finally, we mention that Jessup's result [Je,Prop.3.6] also shows that $\operatorname{cat}_{0}(E) \geq \operatorname{cat}_{0}(B)+1$ for any fibration in which $F$ is positively elliptic - without the hypothesis of TNCZ. These latter observations give some interesting complements to the results presented here.

\section{References}

[Au] M. A ubry, Homotopy Theory and Models, DMV Seminar 24 (1995), Birkhäuser, Basel.

[Ba] H. J. B aues, Algebraic Homotopy, Cambridge Tracts in Mathematics, vol. 15, Cambridge University Press, Cambridge, 1989.

[Co] O. Cornea, There is Just One Rational Cone-Length, Transactions A. M. S. 344 (1994), 835-848.

[Co-Fé-Le] O., Cornea, Y. Félix and J.-M. Lemaire, Rational Category and Cone Length of Poincaré Complexes, Topology 37 (1998), 743-748.

[D-G-M-S] P., Deligne, P. Griffiths, J. Morgan and D. Sullivan, Real Homotopy Theory of Kähler Manifolds, Invent. Math. 29 (1975), 245-274.

[Fé] Y. Félix, La Dichotomie Elliptique-Hyperbolique en Homotopie Rationnelle, Astérisque 176 (1989).

[Fé-Ha 1 Y. Félix and S. Halperin, Formal Spaces with Finite-Dimensional Rational Homotopy, Transactions A. M. S. 270 (1982), 575-588.

[Fé-Ha $\left.\mathrm{Ha}_{2}\right] \quad$ Y. Félix and S. Halperin, Rational L-S Category and its Applications, Transactions A. M. S. 273 (1982), 1-37.

[Fé-Ha-Le] Y. Félix, S. Halperin and J.-M. Lemaire, The Rational LS Category of Products and of Poincaré Duality Complexes, Topology 37 (1998), 749-756.

[Fé-Th] Y. Félix and J.-C. Thomas, The Monoid of Self-Homotopy Equivalences of Some Homogeneous Spaces, Expositiones Math. 12 (1994), 305-322.

[Gr-Mo] P. Griffiths and J. Morgan, Rational Homotopy Theory and Differential Forms, Progress in Mathematics, vol. 15, Birkhäuser, Boston 1983. 
[Ha 1 S. Halperin, Finiteness in the Minimal Models of Sullivan, Transactions A. M. S. 230 (1977), 173-199.

[Ha $\left.\mathrm{Ha}_{2}\right]$ S. Halperin, Rational Fibrations, Minimal Models and Fiberings of Homogeneous Spaces, Transactions A. M. S. 244 (1978), 199-223.

[Ha 3 S. Halperin, Lectures on Minimal Models, Mem. S. M. F. 9/10 (1983).

[Ha-St] S. Halperin and J. Stasheff, Obstructions to Homotopy Equivalences, Advances in Math. 32 (1979), 233-279.

[He] K. Hess, A Proof of Ganea's Conjecture for Rational Spaces, Topology 30 (1991), $205-214$.

[Ja] I. J a mes, Lusternik-Schnirelmann Category, Handbook of Algebraic Topology, Elsevier, 1995, pp. 1293-1310.

[Je] B. Jessup, Rational L-S Category and a Conjecture of Ganea, J. of Pure and Appl. Alg. 65 (1990), 57-67.

[Lu] G. Lupton, Note on a Conjecture of Stephen Halperin, Springer Lecture Notes in Mathematics, vol. 1440, 1990, pp. 148-163.

[McC] J. Mc Cleary, User's Guide to Spectral Sequences, Mathematics Lecture Series, vol. 12, Publish or Perish, Wilmington, 1985.

[Ma] M. Mark l, Towards One Conjecture on Collapsing of the Serre Spectral Sequence, Rend. Circ. Mat. Palermo (2) Suppl. 22 (1990), 151-159.

[Me] W. Meier, Rational Universal Fibrations and Flag Manifolds, Math. Ann. 258 (1983), 329-340.

[Sh-Te] H. Shiga and M. Tezuka, Rational Fibrations, Homogeneous Spaces with Positive Euler Characteristic and Jacobians, Ann. Inst. Fourier 37 (1987), 81-106.

[Ta] D. Tanré, Homotopie Rationnelle: Modèles de Chen, Quillen, Sullivan, Springer Lecture Notes in Mathematics, vol. 1025, 1983

[Th 1 J.-C. Thomas, Rational Homotopy of Serre Fibrations, Ann. Inst. Fourier 31 (1981), 71-90.

$\left[\mathrm{Th}_{2}\right] \quad$ J.-C. Th o m a s, Eilenberg-Moore Models for Fibrations, Transactions A. M. S. 274 (1982), 203-225.

[Vi] M. Vigué, Réalisation de Morphismes Donnés en Cohomologie et Suite Spectrale d'Eilenberg-Moore, Transactions A. M. S. 265 (1981), 447-484. 